\title{
LA PLANIFICACIÓN CENTRADA EN LA PERSONA: UN EJEMPLO DE BUENA PRÁCTICA EN EL ÁMBITO DE LA DISCAPACIDAD INTELECTUAL
}

\author{
Araceli Arellano Torres \\ Feli Peralta López \\ Universidad de Navarra
}

\begin{abstract}
RESUMEN: En la actualidad, el foco de la planificación de los apoyos en el ámbito de la discapacidad intelectual es la persona, poniendo un especial énfasis en la toma de decisiones de los usuarios de los diferentes servicios y, por tanto, en la necesidad de consultarles. En ese sentido, la Planificación Centrada en la Persona (PCP) se convierte en un ejemplo de buena práctica que toma como referente central a la persona, con la pretensión de llegar a conocer lo que es deseable para ella y hacerlo posible. Para ello, es preciso que los profesionales, participen y se impliquen en los cambios que esta buena práctica requiere.

En este artículo se presenta un cuestionario dirigido a valorar los conocimientos de los profesionales, que trabajan en centros de atención a personas con discapacidad, acerca de la PCP. Se describen los resultados de un estudio piloto $(\mathrm{N}=18)$, cuyos resultados señalan la importancia de formar, e informar, a los profesionales, para que sean capaces de asumir propuestas que tomen como foco de atención a la persona. Una organización que verdaderamente apueste por esta metodología, será más eficaz y funcional si implica a quienes tienen que ponerla en marcha, ofreciéndoles formación acerca de esta metodología de trabajo.
\end{abstract}

PALABRAS CLAVE: Discapacidad intelectual, planificación centrada en la persona (PCP), profesionales, buenas prácticas.

\section{PERSON-CENTRED PLANNING: AN EXAMPLE OF BEST PRACTICES IN THE FIELD OF INTELLECTUAL DISABILITY}

\footnotetext{
ABSTRACT: At present, the focus of supports in the field of intellectual disability is the individual, with special emphasis on decision-making by users of different services and, therefore, on the need of consultation. In that sense, Person Centered Planning (PCP) becomes an example of best practice that
} 
leads the individuals as a central reference, in order to know what is desirable for them and make it possible. This practices requires the professional's participation and involvement, to carry out all the changes that it implies. The purpose of this paper is to present a questionnaire, which is used to assess the perception of professionals on PCP methodology. The results of a pilot study $(N=18)$ are described, showing the importance of professional's training. An organization that is really committed to this approach, will be more effective if it involves those who have to implement it, providing them training related to this best practice.

KEYWORDS: Intellectual disability, person centred planning, professionals, best practices.

Recibido: 20/04/2015

Aceptado: 24/07/2015

Correspondencia: Araceli Arellano Torres, Departamento de Aprendizaje y Curriculum, Facultad de Educación y Psicología, Campus Universidad de Navarra, Edificio de Bibliotecas, 31009 Pamplona. Email: aarellanot@unav.es.

\section{La PCP COMO ejemplo de bUena práctica}

La Planificación Centrada en la Persona (de aquí en adelante, PCP) es considerada una referencia de buena práctica en el ámbito de los servicios socioeducativos, dirigidos a las personas con discapacidad intelectual (de aquí en adelante, $\mathrm{DI}^{1}$ ), desde la segunda mitad del siglo XX hasta la fecha. Tanto es así que esta filosofía y método de trabajo podría situarse a la altura de logros como el paradigma de la inclusión, la desinstitucionalización, el modelo de apoyos basado en la calidad de vida o el movimiento de autodeterminación de las personas con discapacidad (Brown, Parmenter y Percy, 2007).

Concretamente, en las últimas décadas, la personalización de los servicios y apoyos emerge con fuerza como un medio para responder a las necesidades de los usuarios, para promover su autonomía, su participación y su capacidad para tomar decisiones en áreas significativas de su vida (como por ejemplo, relaciones sociales, metas y objetivos personales, proyecto de vida, participación en la comunidad, preferencias educativas o profesionales, etc.) (Coyle, 2011; Pallisera, 2011; O'Brien, y Lovett, 1992). En este proceso de transformación de los servicios, surgen nuevos métodos que pretenden dar voz a la persona en los procesos de intervención, teniendo en cuenta su historia, sus deseos y sus expectativas en cuanto a la elaboración

1. Si bien la PCP es un método y una filosofía aplicable a todas las personas con o sin discapacidad, en este artículo nos centraremos en el grupo específico de personas con discapacidad intelectual, basándonos en la definición de la AAIDD (2012): son personas con "limitaciones significativas tanto en el funcionamiento intelectual como en la conducta adaptativa tal, que se manifiestan en habilidades conceptuales, sociales y prácticas" (AAIDD, 2012). El término DI se ha sustituido en el DSM 5 (APA, 2013) por "trastornos del desarrollo intelectual". 
de su plan de futuro personal. Así pues, desde aproximadamente mediados de los 80, las organizaciones dedicadas a la atención de la discapacidad en general (y en particular de la discapacidad intelectual), han tomado como referente central a la persona con la pretensión de llegar a conocer lo que es deseable para ella y trabajar en su consecución, es decir, tratar de "hacer lo deseable posible". De este modo, el objetivo de la planificación de los recursos y apoyos socioeducativos ha pasado a ser la persona, poniéndose un mayor énfasis en la toma de decisiones de los usuarios o clientes y, por tanto, en la necesidad de consultarles (Brown, Galambos, Poston y Turnbull, 2007; Holburn, 2003; Holburn y Jacobson, 2004; Wehmeyer y otros, 2007; Wehmeyer y Little, 2013).

La PCP engloba y representa esta nueva forma de trabajar, no solo con una atractiva filosofía; sino sobre todo como una buena práctica -efectiva y real- que permita a profesionales y personas con DI trabajar juntos para convertir los principios de normalización e inclusión en una realidad (Holburn, Gordon y Vietze, 2007; O’Brien y Lovett, 1992). Supone, por lo tanto, un modo diferente de plantear el diseño de los apoyos, al basarse en el punto de vista de las propias personas con discapacidad, yendo más allá del rol de meras espectadoras al que, hasta hace poco, eran asignadas.

\section{Fundamentos de la PCP}

En este trabajo, teniendo en cuenta la revisión de la literatura (Cambridge y Carnaby, 2005; Coyle, 2011; López, Marín y de la Parte, 2004; Mata y Carratalá, 2007; Pallisera, 2011), entendemos la PCP como un proceso de colaboración que pretende que la persona con DI, junto con un grupo de apoyo, formule sus propios planes y metas de futuro (su plan de vida deseado), así como los medios, estrategias y acciones necesarias para conseguir esos logros. El propósito de la PCP es alcanzar cuatro principios clave: derechos, independencia, elección e inclusión (Cambridge y Carnaby, 2005; Mata y Carratalá, 2007). Así, a través de la acción compartida y la mutua interdependencia, entre usuarios y profesionales, la PCP pretende conseguir que las personas con discapacidad: elijan el estilo de vida que prefieran; sean más independientes; se conviertan en miembros activos de la comunidad; fortalezcan el grado de conexión con personas importantes para ellas; expresen sus preferencias y deseos; y realicen elecciones (Holburn, 2003; Holburn, y Vietze, 2002; Sevilla y otros, 2009).

A pesar de la diversidad en cuanto a la aplicación de la PCP, existe un acuerdo entre los expertos acerca de los principios de esta filosofía y buena práctica (sintetizados en la Tabla 1). Como describen autores como Morgan y Yoder (2012) y Olsson, Jacobson Ung, Swedberg y Ekman (2012), la PCP es, ante todo, un enfoque respetuoso, holístico (no reduce a la persona a su discapacidad o trastorno), individualizado y que persigue la capacitación de la persona. Son precisamente dichos rasgos fundamentales los que deberían ser conocidos, comprendidos y asumidos por parte de los profesionales de los servicios. En el estudio piloto presentado en páginas posteriores, intentaremos abordar el conocimiento que los profesionales encuestados tienen sobre la PCP, ya que, como veremos, condiciona en gran medida el éxito de dicha práctica. 
Tabla 1. Fundamentos de la PCP

\begin{tabular}{|c|c|}
\hline \multicolumn{2}{|r|}{ Una visión general sobre la PCP } \\
\hline Principios & $\begin{array}{l}\text { - Tiene como meta fortalecer la conexión y participación de la persona } \\
\text { en su comunidad } \\
\text { - Promueve apoyos para que la persona tome decisiones, realice } \\
\text { elecciones, exprese sus metas y preferencias, y asuma el control sobre } \\
\text { su vida } \\
\text { - Es un medio para reducir el aislamiento, la segregación y la falta de } \\
\text { oportunidades para la participación de la persona con DI }\end{array}$ \\
\hline Metodología & $\begin{array}{l}\text { - Plantea un nuevo "lenguaje" para la intervención, más accesible y } \\
\text { dinámico } \\
\text { - Se sirve de múltiples herramientas (Personal Histories, Maps, Paths, } \\
\text { Essential Life Planning...) } \\
\text { - Exige una constante búsqueda de formas de expresión y comunicación } \\
\text { que faciliten a la persona con discapacidad mostrar aquellas cosas que } \\
\text { son importantes para ella }\end{array}$ \\
\hline $\begin{array}{c}\text { Rol de } \\
\text { profesionales }\end{array}$ & $\begin{array}{l}\text { - Se convierten en guías y facilitadores del proceso } \\
\text { - Abandonan su rol como responsables únicos de la toma de decisiones } \\
\text { - Requieren de un proceso de formación y sensibilización específico }\end{array}$ \\
\hline
\end{tabular}

Algunas de las preguntas que han guiado esta pequeña investigación son: ¿cómo se concretan estos principios fundamentales?, ¿qué es lo que todo profesional debería tener en cuenta antes de emprender procesos de innovación de PCP? En primer lugar, como ya se ha venido comentando, la PCP se articula en los principios de normalización, inclusión y autodeterminación, que son, a su vez, dimensiones centrales de calidad de vida individual ${ }^{2}$. En ese sentido, las metodologías de la PCP proporcionan a las personas con discapacidad oportunidades para ejercer control en áreas significativas para ellas, como ya se ha mencionado. En otras palabras, les permite convertirse en actores principales, o agentes causales, de su propia vida (Holburn y Vietze, 2002; O'Brien y O'Brien, 2002; Wehmeyer y otros, 2011). En segundo lugar, la PCP es un conjunto de metodologías flexibles y abiertas con amplias posibilidades de aplicación. En ese sentido, una PCP puede durar toda una vida o bien estar centrada en un objetivo concreto a corto plazo; no está limitada a un grupo específico de personas que tengan un determinado nivel de competencia o de edad, ni con una modalidad fija de comunicación, entre otras. Se podría decir que, en cierta manera, la PCP es una metodología que asume una política de "rechazo cero"; es decir, ofrece un servicio a personas con múltiples diagnósticos; de diversas edades y en contextos muy variados. En este artículo, nos centraremos en un grupo específico de personas con $\mathrm{DI}$, desde el ámbito de los servicios so-

2. Para analizar con más detalle la estrecha relación entre dichas dimensiones centrales de bienestar y metodologías que sitúan en el centro a la persona y sus derechos fundamentales (derecho a decidir por sí misma, a elegir, a expresar preferencias, etc.), se pueden consultar fuentes como Schalock y otros (2002), Verdugo, Navas, Gómez, y Schalock (2012), Verdugo y Schalock (2013), Verdugo y otros (2014, 2015), Schalock (2004), Seo y otros (2012), Wehmeyer y Little (2013), entre otros. 
cioeducativos. De hecho, y aunque no nos detengamos en este punto, la filosofía y metodología de la PCP ha sido también asumida en los entornos escolares (sobre todo, centros específicos), a la hora de desarrollar los currícula adaptados a las necesidades especiales de los alumnos con DI.

Finalmente, Ilevar cabo esta metodología supone que los profesionales abandonen términos estrictamente técnicos y aboguen por una presentación de los planes y programas de un modo más atractivo, dinámico y creativo. En cualquier proceso de PCP se requiere el uso de recursos gráficos y apoyos visuales como fotografías, mapas, recortes, dibujos, etc., todos ellos destinados a abrir nuevas formas de comunicación que faciliten a la persona con discapacidad expresar aquellas cosas que son importantes para ella. Las herramientas específicas con las que materializar la PCP son variadas, flexibles y complementarias entre sí (ver, por ejemplo: Black, Mcconkey, Roberts y Ferguson, 2010; Novak y McBride, 2001; Arteta y otros, 2012; Pearpoint, O'Brien y Forest, 2008).

Ahora bien, para que este tipo de propuestas sean efectivas se requiere un serio replanteamiento de las prácticas habituales (tradicionales) dirigidas a las personas con DI. Para ello, es preciso contar con el compromiso de quienes tienen que planificar y facilitar este tipo de acciones: los profesionales. Es precisamente, la reflexión sobre el rol de los profesionales así como sobre su nivel de formación y compromiso hacia la PCP, el punto de partida del estudio piloto presentado en este artículo. Como muestra la investigación de los últimos años, el aumento de la presencia y participación de la persona con DI, y la transformación de los roles de profesionales y servicios de apoyos son pasos fundamentales si queremos avanzar hacia la plena inclusión (Batanero, 2006; Sandoval, 2008).

\section{Proceso de la PCP: el rol del facilitador}

Resulta evidente que una buena práctica que tome como centro a la persona y promueva su inclusión y su autodeterminación, requiere un profundo cambio en la manera de concebir y enfocar los procesos de intervención. Supone, de alguna manera, superar ciertos enfoques rígidos en el trabajo del docente o personal de apoyo, frecuente en el contexto de la discapacidad (Sandoval, 2008). Dicho cambio va a depender en gran medida de la implicación y preparación de los profesionales y, por ello, de sus conocimientos, creencias, percepciones y actitudes acerca de esta metodología o enfoque que deben ser puestos en marcha (Morgan y Yoder, 2012).

Como vemos reflejado en la Figura 1, la PCP se inicia siguiendo un proceso cíclico que consta de diversas tareas o pasos: (a) la definición de un círculo de apoyo (personas significativas en la vida del protagonista), (b) la elección de un facilitador (normalmente, un profesional encargado de guiar todo el proceso), (c) el desarrollo de un plan de futuro y $(d)$ el seguimiento continuo (que permite ajustar los planes y estrategias a las necesidades de la persona).

La PCP requiere reorientar las prácticas socioeducativas hacia un nuevo enfoque que, entre otras cosas, transforma de manera significativa el rol de los expertos y de la persona con DI, a lo largo de todo el proceso. Como se ha venido reflejando, la PCP tiene como premisa básica la idea de que el poder debe trasladarse de quienes proporcionan los servicios hacia quienes los reciben (Holburn, 2003). En ese sentido, 
es imprescindible que el profesional, elegido facilitador, comprenda y comparta los fundamentos, principios y procedimientos de la PCP y mantenga una buena relación con la persona central. Dentro de su papel de facilitador lo fundamental no es tanto el logro de los objetivos, como la dinamización del propio proceso.

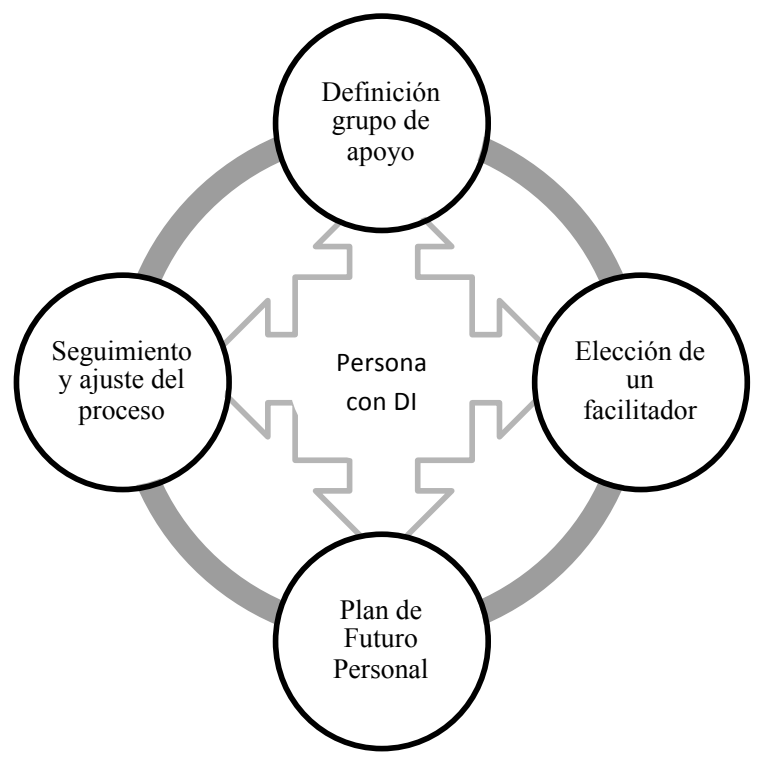

Figura 1. Proceso de la PCP

En definitiva, la implicación de los profesionales es clave, hasta el punto de que algunos investigadores han identificado una mala facilitación como uno de los obstáculos más importantes para que la PCP sea efectiva (Novak y McBride, 2002; Holburn, 2003; Robertson y otros, 2007a; Robertson y otros, 2007b). Es por eso por lo que este artículo tiene como propósito indagar en cuestiones como las siguientes: ¿Conocen los profesionales los rasgos principales de la PCP?, ¿valoran los beneficios que esta metodología puede aportar en su área de trabajo?, ¿han recibido formación al respecto?, ¿cuáles son las dificultades que, mayoritariamente, se encuentran a la hora de trabajar según esta buena práctica? Para ahondar en estos interrogantes, presentamos en este artículo un estudio que tiene su origen en un Trabajo Fin de Máster (Máster en Intervención Educativa y Psicológica) ${ }^{3}$, cuyos objetivos principales se concretan en: (1) presentar un cuestionario que pueda ser útil para valorar los conocimientos, creencias y actitudes de los profesionales sobre la PCP; y (2) describir los resultados preliminares de su aplicación en un estudio piloto. Es, por tanto, un trabajo descriptivo, meramente exploratorio, pero que puede abrir nuevas líneas de investigación, en cuanto a mejorar las formas de valorar conocimientos y necesidades de los profesionales respecto a la PCP.

3. La autora de este trabajo, dirigido por Felisa Peralta fue Poy López de Dicastillo. 


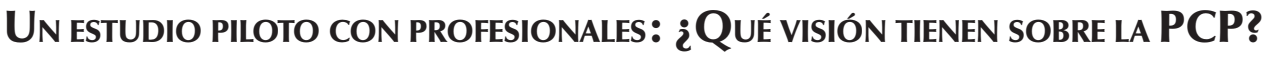

\section{Participantes}

Para la realización de este estudio, se invitó a 30 profesionales de un centro de la comarca de Pamplona, de los cuales 18 se prestaron a participar voluntariamente 4 . En cuanto a la descripción de la muestra (ver Tabla 2): el 33\% son hombres y el 67\% mujeres. La mayoría tienen entre 30 y 40 años (56\%), el 28\% entre 41 y 50 años, sólo el $17 \%$ tiene más de 50 . En lo que respecta a la titulación académica, más de la mitad son diplomados (56\%), un 6\% licenciados, y un 17\% cursó estudios de Formación Profesional (el porcentaje restante señala no tener estudios o no contesta). En referencia a la trayectoria profesional, la mitad de la muestra dice haber trabajado menos de 20 años en educación especial y el 39\%, cifra nada despreciable, entre 21 años y 30. Es relevante el hecho de que el $48 \%$ trabaje asiduamente con personas con DI de grado profundo, y el $45 \%$ lo haga con personas con grado severo. Sólo el 7\% trabaja con personas con grado moderado.

Tabla 2. Características de la muestra participante en el estudio $(\mathrm{N}=18)$

\begin{tabular}{|c|c|c|c|c|}
\hline & & & \\
\hline & & $\mathbf{F r}$ & $\%$ & Total \\
\hline \multirow{2}{*}{ Sexo } & Masculino & 6 & 33,3 & \multirow{2}{*}{18} \\
\hline & Femenino & 12 & 66,7 & \\
\hline \multirow{3}{*}{ Edad } & $31-40$ & 10 & 55,6 & \multirow{3}{*}{18} \\
\hline & $41-50$ & 5 & 27,8 & \\
\hline & $>50$ & 3 & 16,7 & \\
\hline \multirow{5}{*}{ Titulación académica } & F.P & 3 & 16,7 & \multirow{5}{*}{18} \\
\hline & Diplomatura & 10 & 55,6 & \\
\hline & Licenciatura & 1 & 5,7 & \\
\hline & Otros & 3 & 16,7 & \\
\hline & $\mathrm{NC}$ & 1 & 5,7 & \\
\hline \multirow{4}{*}{$\begin{array}{c}\text { Años trabajados en Educación } \\
\text { Especial }\end{array}$} & $<20$ & 9 & 50 & \multirow{4}{*}{18} \\
\hline & $>21$ & 7 & 38,9 & \\
\hline & $>30$ & 1 & 5,7 & \\
\hline & NC & 1 & 5,7 & \\
\hline \multirow{5}{*}{$N^{\circ}$ de horas de trabajo semanales } & $<20$ & 1 & 5,7 & \multirow{5}{*}{18} \\
\hline & $>21$ & 1 & 5,7 & \\
\hline & $>30$ & 5 & 27,8 & \\
\hline & $>39$ & 8 & 44,4 & \\
\hline & Variable & 3 & 16,7 & \\
\hline \multirow{4}{*}{$\begin{array}{l}\mathbf{G}^{\circ} \text { de discapacidad de las } \\
\text { personas con las que trabaja } \\
\text { (criterios DSM-IV) }\end{array}$} & Ligera & 0 & 0 & \multirow{4}{*}{29} \\
\hline & Moderada & 2 & 6,9 & \\
\hline & Severa & 13 & 44,82 & \\
\hline & Profunda & 14 & 48,3 & \\
\hline
\end{tabular}

4. Agradecemos la colaboración de los profesionales del centro Oncineda, así como la disposición por parte de su director para la participación en este estudio. 


\section{Instrumento}

Se diseñó un cuestionario ad hoc para la obtención de los datos. Para su elaboración se tomaron como referencia otros métodos empleados en investigaciones previas dentro de este campo de estudio (ver, por ejemplo, Grigal, Neubert, Moon y Graham, 2003; Wehmeyer, Agran y Hughes, 2000), así como la propia fundamentación teórica de esta metodología y filosofía. El objetivo de este estudio es explorar en qué medida puede ser útil el uso de una herramienta como esta para acceder a los conocimientos de los profesionales de los servicios acerca de la PCP (como un primer paso para elaborar propuestas de formación).

El cuestionario empleado consta de 25 ítems enunciados en forma bien de preguntas abiertas (ítem 1) o bien de preguntas cerradas (ítems 2 a 25). La pregunta abierta contenida permite que los profesionales describan con sus propias palabras qué entienden por Planificación Centrada en la Persona. Las preguntas cerradas se han formulado de acuerdo a las principales características de la PCP según la literatura más relevante, citada en páginas anteriores. Los formatos de respuesta para dichas preguntas son, por una parte, elección entre varias opciones dadas (se han introducido algunas afirmaciones erróneas o tópicos como distractores) y, por otra, escala tipo Likert de cinco puntos $(1=$ Totalmente en desacuerdo/Nada, 2= En desacuerdo/Poco, 3= Ni de acuerdo, ni en desacuerdo/Regular, 4= De acuerdo/Bastante, 5= Totalmente de acuerdo/Mucho).

Los ítems del cuestionario se estructuran en torno a cinco secciones (además de un apartado previo con información demográfica de la muestra, que pudiera servir como variables de corte para el análisis de datos): (1) Conocimiento sobre la PCP y sus características (ítems 1 a 3); (2) Creencias y opiniones sobre la PCP en su conjunto (ítems 4 a 20); (3) Estrategias que promueven la PCP y grado de utilización (ítems 21 a 23); (4) Formación en este campo: grado y tipo de formación recibida (ítem 24) y; (5) Dificultades para el desarrollo o aplicación de la PCP (ítem 25).

Se presenta en el Anexo 1 el cuestionario completo (en su versión piloto) que, como decimos, queda pendiente de revisión. A la vista de los datos obtenidos, se prevé mejorar dicho instrumento y realizar un estudio psicométrico con una muestra más amplia, con el fin de lograr, si es el caso, una versión definitiva.

\section{Análisis de datos}

El proceso de análisis nos ha permitido reflejar las respuestas del total de los profesionales participantes $(\mathrm{N}=18)$ en frecuencias y porcentajes. Los datos provenientes de las preguntas cerradas fueron, primero, codificados y posteriormente tabulados y analizados. Dado el tamaño de la muestra no se consideró adecuado ir más allá de este análisis exploratorio. El contenido de las preguntas abiertas, por su parte, fue también codificado y la valoración de las categorías resultantes completó los datos obtenidos de las escalas de evaluación.

\section{Resultados y discusión}

Se presenta, a continuación, un análisis descriptivo de los datos obtenidos en este estudio piloto, según las categorías principales del cuestionario. 


\section{Conocimientos}

Autores como Holburn (2003), Pallisera (2011), Robertson y otros (2007a, 2007b), advierten que el desconocimiento de las posibilidades de este modelo o metodología es uno de los obstáculos más importantes para su implantación. Por eso, es clave valorar en qué medida los profesionales conocen, al menos en sus principios fundamentales, la PCP. En este caso, los conocimientos que los profesionales encuestados tienen acerca de dicha metodología son imprecisos y generales, más teóricos que prácticos.

Así, al pedirles que definieran con sus palabras la PCP, casi la mitad de los encuestados (44\%) la describen y/o asemejan al Plan de Atención Individualizado (PAI), siendo este un término más familiar para este colectivo. Este dato es importante ya que, metodológicamente hablando, en los PAI se establecen los objetivos y se planifican las intervenciones en función de la disponibilidad de servicios y recursos, en lugar de en función de las preferencias individuales de la persona (López, Marín y De la Parte, 2004). Por otra parte, un 22\% recoge la idea de que la PCP es un "plan para la persona basado en sus motivaciones e intereses". El resto de los profesionales o no contesta o da un respuesta no pertinente, por lo que inferimos, en estos casos, falta de conocimiento o inseguridad en su visión acerca de la PCP.

En cuanto a los medios que facilitan la puesta en marcha y consecución de la PCP en su contexto: un $78 \%$ de los profesionales señala "el conocimiento de las necesidades de la persona"; un 44\% "escuchar y comprender a la persona" y un 39\% "mostrar actitud colaborativa y de interdependencia". Un 33\% de los encuestados marca el "cambio de creencias erróneas" como un medio importante para llevar a cabo la PCP, siendo este aspecto el menos significativo para ellos, por lo tanto. No obstante, y como es sabido por la investigación previa (Pajares, 1992) las creencias condicionan y guían los modos y estilos de actuación, ya que conocimientos, creencias y práctica se retroalimentan. Al contrario de lo que parecen pensar los participantes en este estudio, la puesta en marcha de este enfoque, requiere también un cambio profundo en las prácticas y creencias habituales de los profesionales (Pallisera, 2011). Por otra parte, el uso de metodologías innovadoras como esta, a su vez, modifica la visión de los profesionales, que se muestran más sensibilizados hacia las necesidades y derechos de las personas a las que apoyan (Novak y McBride, 2002; Holburn, 2003; Holburn y Jacobson, 2004), por lo que podemos pensar que una vez se implican en procesos de PCP, el cambio de creencias se inicia.

Respecto a las dimensiones que caracterizan a la PCP, constatamos que la mayoría de los encuestados entiende la autodeterminación (o alguno de sus elementos) como un valor fundamental de la PCP. Lo podemos ver reflejado en sus elecciones, recogidas en la Tabla 3 .

Como hemos mencionado, podemos afirmar que estos profesionales tienen un conocimiento genérico sobre las dimensiones o conductas asociadas a la PCP. Ahora bien, estas dimensiones se han seleccionado más como resultado que como proceso y dicen poco sobre cómo se hace o cómo se logra (por ejemplo, no aparece seleccionada la dimensión "aumentar sus experiencias" que implica directamente al profesional). Cabe advertir al respecto que sólo cuando las personas con DI tienen la oportunidad de practicar conductas autodeterminadas, convenientemente 
facilitadas por sus apoyos (sean naturales o formales), serán menos dependientes y alcanzarán mayores cotas de calidad de vida, inclusión y participación social (Batanero, 2006; López, Marín y de la Parte, 2004; Wehmeyer, Agran y Hughes, 2000; Wehmeyer y Litlle, 2013).

Tabla 3. Respuestas al ítem sobre indicadores de la PCP

\begin{tabular}{|l|c|c|}
\hline \multicolumn{1}{|c|}{ La PCP puede referirse a la capacidad de la persona con DI para... } \\
\hline \multicolumn{1}{|c|}{ Opciones } & Frecuencia & Porcentaje \\
\hline - elegir el estilo de vida que prefiera & 9 & $50 \%$ \\
- ser más independiente & 11 & $61 \%$ \\
- ser miembro activo de la comunidad & 10 & $55 \%$ \\
- hacer cosas solo/a, siempre sin ayuda & 2 & $11 \%$ \\
- solicitar los apoyos que precisa & 12 & $66 \%$ \\
- establecer metas en la vida & 10 & $55 \%$ \\
- resolver los problemas sin contar con otros & 1 & $5 \%$ \\
- expresar intereses, preferencias y opiniones & 10 & $55 \%$ \\
- tomar sus propias decisiones & 10 & $55 \%$ \\
- aumentar sus experiencias & 11 & $61 \%$ \\
- construir relaciones & 9 & $50 \%$ \\
\hline
\end{tabular}

Creencias sobre la PCP

De acuerdo con Borg (2001), las creencias son proposiciones, conscientes o inconscientes, que denotan un compromiso con valores y tienen una importante relación con la conducta en cuanto que están imbuidas de un compromiso emotivo que disponen o guían el pensamiento y la acción del individuo. Esto es, las creencias y actitudes de los profesionales tienen una profunda influencia en la práctica socioeducativa y en su deseo o no de revisarla.

A este respecto, se presentan a los profesionales 17 ítems que reflejan distintas creencias acerca de la PCP (favorables a este proceso o bien consideradas obstáculos), de acuerdo con los principios teóricos recogidos en la primera parte de este artículo (la PCP como proceso colaborativo, que respeta los intereses y metas de la persona, que requiere un cambio profundo de las prácticas, etc.).

En este estudio piloto, las creencias con las que los profesionales encuestados expresan un alto nivel de acuerdo (marcan las opciones De acuerdo o Muy de acuerdo) son las siguientes: la importancia de contar, a lo largo de este proceso, con la red social de apoyos de la persona (100\%); y la necesidad, por otra parte, de implicar también a la familia (89\%). Destacamos este hecho como un elemento muy positivo, dado que, como explica Pallisera (2011), ambos agentes necesariamente deben ser participantes activos en la PCP, constituyendo el círculo de apoyo de la persona. A este respecto, un $33 \%$ está de acuerdo, o muy de acuerdo, con que la PCP puede desarrollarse sin que 
la persona conozca el proceso que se va a llevar a cabo (ítem 20), algo que resulta Ilamativo dado que es este, precisamente, el principio fundamental de la PCP: situar en el centro a la persona con $\mathrm{DI}$, haciéndole partícipe y protagonista de dicho proceso.

Por otra parte, los encuestados se muestran de acuerdo (señalan las opciones De acuerdo o Muy de acuerdo) con que el proceso de la PCP mejora las posibilidades de inserción social de las personas con DI (100\%); conlleva respetar su dignidad $(100 \%)$; y exige a los servicios un cambio en su estructura (77\%). Relacionado con esto último, la necesidad de transformación, una pequeña parte de la muestra (22\%) refiere estar de acuerdo o muy de acuerdo con el ítem "Promover la PCP conlleva cierto caos en los servicios y falta de control por parte de los profesionales". Este es quizá uno de los obstáculos más importantes para implementar procesos de PCP: la falsa creencia según la cual otorgar mayor control a la persona con DI tiene consecuencias negativas sobre el rol de los profesionales y servicios (Novak y McBride, 2002; Robertson y otros, 2007a). Entendemos que esta idea puede estar influida por otras como, por ejemplo, que el $56 \%$ de la muestra considere que, como profesionales, carecen de recursos suficientes para llevar a cabo este proceso (están de acuerdo o muy de acuerdo).

A pesar de las dificultades percibidas, según el $67 \%$ de los participantes en el estudio, las personas con DI tienen suficientes oportunidades para alcanzar sus metas, incluso para el $89 \%$ la persona con grave DI también puede participar en procesos de este tipo. Sin embargo, ante la afirmación "Para llevar a cabo la PCP se precisa que la persona tenga un cierto nivel de capacidad", los encuestados se muestran divididos en sus respuestas (algo paradójico teniendo en cuenta sus respuestas previas): un 50\% afirma estar poco de acuerdo, o en desacuerdo; un $28 \%$ ni de acuerdo ni en desacuerdo; y un $22 \%$ de acuerdo o muy de acuerdo.

\section{Estrategias para llevar a cabo la PCP}

Sobre el grado en que aplican las estrategias recogidas en el cuestionario, el 61\% dice dedicar bastante o mucho tiempo a conocer los intereses de la persona de cara a desarrollar las diferentes actividades del día, un $11 \%$ dedica más bien poco tiempo a este menester y el $22 \%$ reconoce hacerlo poco. Cabe recordar que, según la revisión hecha por Dowling, Manthorpe y Cowley (2007), acerca de la investigación en este ámbito, es precisamente dedicar tiempo a conocer a cada persona, teniendo en cuenta sus necesidades, deseos e intereses únicos, uno de los factores que compromete el éxito de la PCP.

Asimismo, un 50\% reconoce que los usuarios de su centro carecen de suficientes oportunidades para elegir o tomar decisiones. Convendría profundizar en las razones de la falta de oportunidades para elegir. Ya Novak y McBride (2002) advertían de la dificultad que tiene para los profesionales otorgar control y poder de decisión a personas que no están acostumbradas a ello (como hemos podido ver en algunas respuestas del bloque previo). Fundamentalmente, el problema que subyace a obstáculos como este es el paso de los roles tradicionales hacia formas de planificación colaborativa. Consideramos preciso profundizar, en futuros estudios, en este apartado sobre estrategias y medios para llevar a cabo la PCP que, indudablemente, está íntimamente relacionado con las creencias y actitudes de los profesionales. 
Formación y dificultades y obstáculos para la PCP

En cuanto a la formación, abordada únicamente en el ítem 24, los profesionales expresan lo siguiente: un 39\% afirma no haber recibido nunca formación específica sobre la PCP; el 28\% poca; el $17 \%$ de forma regular; y el 6\% bastante. Los tipos de formación mencionados por algunos profesionales son: estudios formativos en la universidad, curso básico de PCP y formación interna acerca de esta metodología.

Finalmente, las dificultades percibidas por ellos de cara al desarrollo de la PCP son: en primer lugar, la falta de recursos y de apoyo institucional (72\%), y la falta de formación (50\%) o la falta de tiempo (44\%), en segundo y tercer lugar. Estos factores contribuyen a que la aplicación de este modelo o metodología para dispensar apoyos se realice más lentamente en nuestro contexto que en el ámbito anglosajón (Pallisera, 2011). Ya la investigación previa examina algunas de las barreras frecuentes para llevar a cabo procesos de PCP, destacando, entre otras: la escasa formación de los profesionales, la falta de tiempo, lo costoso de la personalización de los apoyos en entidades con gran número de usuarios, etc. (Morgan y Yoder, 2012; Novak y McBride, 2002; Dowling, Manthorpe y Cowley, 2007). Como explican Magit-McLaughlin, Spinosa y Marsalis (2002), muchos de estos obstáculos se derivan del hecho de querer integrar la PCP en entornos de servicios tradicionales, basados en criterios más rígidos que los que propone esta metodología. De ahí que los nuevos modelos de servicios que genera la PCP requieren una óptima preparación de los profesionales que tienen que aplicarlos a su quehacer socioeducativo y un cambio profundo en las organizaciones que apuesten por este modelo (McConkey y Collins, 2010). Tal y como aprecian Coyle y Collins (2010), es necesario examinar los conocimientos y habilidades de los profesionales, en relación a la PCP, para comprender cómo éstos pueden reducir o eliminar ciertas barreras percibidas en la inclusión social de las personas con DI.

\section{Limitaciones}

Los resultados de este estudio piloto, como ya se ha indicado anteriormente, no se pueden generalizar por las limitaciones metodológicas que presenta: reducido tamaño de la muestra; participación voluntaria y no aleatoria; sesgo en la representación por sexo y por nivel de $\mathrm{Cl}$ de las personas con DI; o ítems no respondidos por la totalidad de los encuestados. Asimismo, y coincidiendo con Pajares (1992), es preciso advertir que las creencias deben ser inferidas de lo que la gente dice, intenta y hace, lo que da cuenta de la dificultad que conlleva su estudio, ya que no siempre coincide lo que uno dice que hace con lo que realmente hace.

A pesar de estas limitaciones y del carácter eminentemente descriptivo de este trabajo, los datos obtenidos, propios de un estudio piloto, ofrecen una aproximación a los conocimientos y creencias que un grupo de profesionales presenta en torno a la PCP, siendo de utilidad a la hora de realizar propuestas para su formación.

\section{Conclusiones}

Según se recoge de la literatura, aplicar nuevas metodologías enfocadas a una buena práctica que tomen como centro a la persona con DI requiere un profundo 
cambio en la manera de concebir y enfocar un servicio y la actividad que genera. Esta transformación va a depender en gran medida de la implicación de los profesionales y, por ello, de sus conocimientos y actitudes acerca de dicha metodología. El análisis de las percepciones de los profesionales puede ser un punto clave para el diseño de las propuestas orientadas al desarrollo y penetración de la PCP en las organizaciones.

La apuesta de una organización por primar objetivos que asuman la filosofía que subyace en la PCP y que se desarrollen de acuerdo con esta metodología, será más eficaz y funcional si se parte del conocimiento previo de sus profesionales acerca del tema (por ejemplo, teorías implícitas que tienen acerca de la PCP, importancia que le otorgan, cómo diseñan su práctica, motivación que tienen por alcanzar esta meta, etc.).

El estudio de las creencias de los profesionales también es un factor importante, ya que cualquier propuesta de innovación metodológica u organizativa conviene comenzarla partiendo del punto de vista previo del que tiene que llevarla a cabo. En lo que a la PCP se refiere, un profesional motivado por favorecerla estará más dispuesto a aprender y practicar las estrategias que conducen a su logro que el que no cree en el potencial de aprendizaje que dichas estrategias tienen para el usuario.

En el estudio piloto aquí presentado, se ha analizado el conocimiento que un grupo de profesionales tienen sobre la PCP y su aplicación, si bien no se ha profundizado en cómo llevan a la práctica sus propuestas.

Será preciso, en futuras investigaciones, mejorar el contenido del instrumento de evaluación, sobre todo en cuanto a la exploración de las actitudes y estrategias, así como ampliar la muestra participante que nos permita obtener más datos al respecto. Por otra parte, una nueva línea de investigación podría centrarse en analizar en qué medida los profesores de contextos escolares y educativos conocen y aplican con sus alumnos esta metodología.

Entendemos que los datos aquí presentados pueden ser destacables, de cara a elaborar programas de formación continua que proporcionen a los profesionales de los servicios un conocimiento más explícito sobre el concepto de la PCP y sus características, junto con una adecuada instrucción práctica en cuanto a las estrategias y materiales a emplear en su quehacer diario como auténticos facilitadores de este proceso.

\section{Referencias Bibliográficas}

American Association of Intellectual and Developmental Disabilities (2011). Discapacidad intelectual: definición, clasificación y sistemas de apoyo (11 ${ }^{\mathrm{a}}$ ed.). Madrid: Alianza.

American Psychiatric Association (2013). Diagnostic and Statistical Manual of Mental Disorder. Fith Edition. DSM-5. Washington: American Psychiatric Publishing.

Arteta, R., Peralta, F., Ezquerro, A., Zazo, M. J., y Barbería, M. (2012). Planificación Centrada en la Persona. Pamplona: ANFAS.

Batanero, J. M. (2006). ¿Educación inclusiva en nuestros centros educativos? Sí, pero ¿cómo? Contextos Educativos, 8(9), 135-145.

Black, L. A., McConkey, R., Roberts, P., y Ferguson, P. (2010). Developing a personcentred support service for families caring for children with severe learning disabilities in rural and urban areas. Journal of Intellectual Disabilities, 14(2), 111-131. 
Borg, M. (2001). Teachers Beliefs. ELT Journal, 55(2), 186-188.

Brown, I., Galambos, D., Poston, D.J., y Turnbull, A.P. (2007). Person centered and family centered support. En I. Brown y M. Percy (Eds.), A comprehensive guide to intellectual and developmental disabilities (pp. 351-361). Baltimore: Paul H. Brookes.

Brown, I., Parmenter, T.R., y Percy, M. (2007). Trends and issues in intellectual and developmental disabilities. En I. Brown y M. Percy (Eds.). A comprehensive guide to intellectual and developmental disabilities (pp. 45-57). Baltimore: Paul H. Brookes.

Cambridge, P., y Carnaby, S. (Eds.) (2005). Person centered planning and care management with people with learning disabilities. Londres: Jessica Kingsley Publishers.

Coyle, D. (2011). Impact of person-centred thinking and personal budgets in mental health services: reporting a UK pilot. Journal of Psychiatric and Mental Health Nursing, 18(9), 796-803.

Dowling, S., Manthorpe, J., y Cowley, S., (2007). Working on person-centred planning. From amber to green light? Journal of Intellectual Disabilities, 11(1), 65-82.

Grigal, M., Neubert, D., Moon, M. S., y Graham, S. (2003). Self-Determination for Students with disabilities: Views of Parents and Teachers. Exceptional Children, $70(1), 97-112$.

Holburn, S. (2003). Cómo puede la ciencia evaluar y mejorar la Planificación Centrada en la Persona. Siglo Cero, Revista Española sobre Discapacidad Intelectual, 34(4), 48-64.

Holburn, S., y Jacobson, J.W. (2004). Implementing and researching person-centered planning. En L. Williams (Ed.), Developmental disabilities: advances in scientific understanding, clinical treatments and community integration (pp. 315-330). Reno, N.V.: Context Press.

Holburn, S., y Vietze, P. (2002). Person-centered planning: Research, practice and future directions. Baltimore: Paul H. Brookes.

López, M.A., Marín, A., y De la Parte, J.M. (2004). La planificación centrada en la persona. Una metodología coherente con el respeto al derecho de autodeterminación. Una reflexión sobre la práctica. Revista Siglo Cero, 35(1), 1-16.

Magit-McLaughlin, D., Spinosa, T.R., y Marsalis, M. (2002). Overcoming the barriers: moving toward a service model that is conductive to person-centered planning. En S. Holburn y P. Veitz (Eds.), Person-centered planning: Research, Practice and Future directions (pp. 127-150). Baltimore: Paul H. Brookes.

Mata, G., y Carratalá, A. (2007). Planificación Centrada en la Persona. Experiencia de la Fundación San Francisco de Borja para personas con DI. Madrid: FEAPSCuadernos de Buenas Prácticas.

Morgan, S., y Yoder, L.H. (2012). A Concept Analysis of Person-Centered Care. Journal of Holistic Nursing, 30(1), 6-15.

McConkey, R., y Collins, S. (2010). Using personal goal setting to promote the social inclusion of people with intellectual disability living in supported accommodation. Journal of Intellectual Disability Research, 54(2), 135-143. 
Novak, A., y McBride, M. (2001). Increasing person-centered thinking: improving the quality of person-centered planning. A manual for person-centered planning facilitators. Minneapolis, Minnesota: University of Minnesota, Institute on Community Integration.

O'Brien, J., y Lovett, H. (1992). Finding a way toward everiday lives. The contribution of person centered planning. Pennsylvania: Office of Mental Retardation.

Olsson, L.E., Jakobsson Ung, E., Swedberg, K., y Ekman, I. (2012). Efficacy of personcentred care as an intervention in controlled trials - a systematic review. Journal of Clinical Nursing, 22, 456-465.

Pajares, M.F. (1992). Teachers Beliefs and Educational research: Cleaning up a messy construct. Review of Educational Research, 62(3), 307-332.

Pearpoint, J., O’Brien, J., y Forest, M. (2008). PATH: a workbook for planning positive possible futures: planning alternative tomorrows with hope for schools, organization, businesses, families ( $2^{\mathrm{a}} \mathrm{ed}$.). Toronto: Inclusion Press.

Peralta, F., González-Torres, M., y Sobrino, A. (2005). Creencias y conocimientos de los profesores acerca de la conducta autodeterminada en personas con discapacidad cognitive. Revista de Investigación Educativa, 23(2), 433-448.

Robertson, J., Hatton, C., Emerson, E., Elliott, J., McIntosh B., Swift, P., y otros. (2007a) Reported Barriers to the Implementation of PCP for People with Intellectual Disabilities in the UK. Journal of Applied Research in Intellectual Disabilities, 20, 297-307.

Robertson, J., Emerson, E., Hatton, C., Elliott, J., McIntosh B., Swift, P., y otros. (2007b) PCP: factors associated with successful outcomes for people with intellectual disabilities. Journal of Intellectual Disability Research, 51(3), 232-243.

Sandoval, M. (2008). La colaboración y la formación del profesorado. Contextos Educativos, 11, 149-159.

Schalock, R.L. (2004). The concept of quality of life: what we know and do not know. Journal of Intellectual Disability Research, 48(3), 203-216.

Schalock, R.L., Brown, I., Brown, R., Cummins, R.A., Felce, D., y otros (2002). Conceptualization, measurement, and application of quality of life for people with intellectual disabilities: report of an international panel of experts. Mental Retardation, 40(6), 457-470.

Seo, H., Wehmeyer, M.L., Palmer, S., Soukup, J.H., Williams-Diehm, K., y otros (2012). Examining individual and environmental factors on levels of self-determination of students with disabilities: The relationship between self-determination and disability categories, gender, and educational settings. The Journal of Special Education: Theory and Practice, 13(2), 1-22.

Sevilla, J., Abellán, R., Herrera, G., Pardo, C., Casas, X., y otros (2009). Un concepto de Planificación Centrada en la Persona para el siglo XXI. Fundación Adapta. Recuperado de: http://www.miradasdeapoyo.org.

Pallisera, M. (2011). La Planificación Centrada en la Persona (PCP): una vía para la construcción de proyectos personalizados con personas con discapacidad intelectual. Revista Iberoamericana de Educación, 56(3), 1-12.

Verdugo, M.A., Gómez, L.E., Arias, B., Navas, P., y Schalock, R.L. (2014). Measuring quality of life in people with intellectual and multiple disabilities: validation of the San Martin Scale. Research in Developmental Disabilities, 35(1), 75-86. 
Verdugo, M.A., Navas, P., Gómez, L., y Schalock, R.L. (2012). The concept of quality of life and its role in enhancing human rights in the field of intellectual disability. Journal of Intellectual Disability Research, 56(11), 1036-1045.

Verdugo, M.A., y Schalock, R.L. (2013). Discapacidad e inclusión: manual para la docencia. Salamanca: Amarú.

Verdugo, M.A., Vicente, E., Fernández-Pullido, R., Gómez-Vela, M., Wehmeyer, M.L., y otros (2015). A psychometric evaluation of the ARC-INICO Self-determination Scale for adolescents with intellectual disabilities. International Journal of Clinical and Health Psychology, 15(2), 149-159.

Wehmeyer, M.L., Abery, B., Zhang, D., Ward, K., Willis, D., Amin, W., y otros (2011). A series of paper on scaling-up efforts to promote self-determination. Personal self-determination and moderating variables that impact efforts to promote selfdetermination. Recuperado de: gsd.org/news/personal-self-determination-andmoderating-variables-impact-efforts-promote-self-determination.

Wehmeyer, M.L., Agran, M., y Hughes, C. (2000). A National survey of teachers` promotion of Self-Determination and Student-Directed Learning. The Journal of Special Education, 34(2), 58-68.

Wehmeyer, M.L., y Little, T.D. (2013). Self-determination, positive psychology, and disability. En. M. L. Wehmeyer (Ed.), Handbook of positive psychology and disability (pp. 116-136). Oxford: Oxford University Press.

Wehmeyer, M.L., Palmer, S., Soukup, J. Garner, N., y Lawrence, M. (2007). Self-determination and student transition planning knowlegde and skills: predicting involvement. Exceptionality, 15, 31-44. 
ANEXO. Cuestionario para valorar perspectiva de los profesionales sobre la PCP (versión piloto, Elaborada por Feli Peralta, dirigida a profesionales de servicios para personas con discapacidad intelectual)

1. ¿Qué entiende por Planificación Centrada en la Persona (PCP)? Describa brevemente este término, o bien, emplee algún sinónimo

2. ¿Qué medio/s considera más adecuado/s para llevar a cabo este proceso con los usuarios de su centro (enumere por orden de importancia)?

- Conocer las necesidades de la persona con DI

- Mantener una actitud colaborativa y de interdependencia (usuario-equipo-familia)

- Cambiar mis creencias erróneas

- Escuchar y comprender a la persona con DI

- Hacer por la persona con DI sólo lo que no puede hacer por sí misma

G Generar actividades que promuevan los intereses de la persona con DI

3. En su opinión, la Planificación Centrada en la Persona (PCP) puede referirse a la capacidad de la persona con DI para... (Puede marcar más de una casilla)

$\square$ elegir el estilo de vida que prefiera

口 ser más independiente

- ser miembro activo de la comunidad

- hacer cosas solo/a, siempre sin ayuda

solicitar los apoyos que precisa

- establecer metas en la vida resolver los propios problemas sin contar con los demás

口 preparar su plan de futuro personal

expresar intereses, preferencias y opiniones

$\square$ tomar sus propias decisiones

$\square$ aumentar sus experiencias

construir relaciones

Manifieste su grado de acuerdo con las CREENCIAS y OPINIONES que se recogen a continuación ( $1=$ Totalmente en desacuerdo; $2=$ En desacuerdo; $3=\mathrm{Ni}$ de acuerdo, ni en desacuerdo; 4= De acuerdo; 5= Totalmente de acuerdo)

4. La PCP consiste en hacer posible el futuro deseable

5. Para llevar a cabo la PCP es preciso modificar los estilos de los profesionales

6. Una persona con grave DI nunca puede participar en este proceso

7. El desarrollo de la PCP implica la participación de la familia

8. Las personas con DI tienen pocas oportunidades de lograr sus propias metas

9. El desarrollo de la PCP requiere un cambio en las estructuras de los servicios

10. Las personas con DI desconocen lo que es mejor para ellas 


\section{(Continuación)}

11. La PCP es un proceso longitudinal (que se realiza a lo largo de la vida, aunque tenga más sentido en la etapa adulta)

12. Los profesionales carecen de recursos para promover la PCP

13. Para llevar a cabo la PCP se precisa que la persona tenga un cierto nivel de capacidad

14. La PCP implica respeto a la dignidad de la persona

15. La persona con DI habitualmente desconoce lo que es mejor para ella

16. Para preparar el plan de futuro de la persona con DI es preciso controlar todo el proceso

17. Trabajar la PCP mejora las posibilidades de inserción social de las personas con DI

18. El desarrollo de la PCP exige contar con una red social de apoyos

19. Promover la PCP conlleva cierto caos en los servicios y falta de control por parte de los profesionales

20. La PCP puede desarrollarse sin que la persona con DI conozca el proceso que se va a llevar a cabo

Conteste las siguientes preguntas ( $1=\mathrm{Nada}, 2=$ Poco, $3=$ Regular, $4=$ Bastante, y $5=$ Mucho $)$

21. ¿Las personas con DI de su institución tienen oportunidad de elegir y tomar decisiones en la medida de sus posibilidades?

22. ¿Considera importante reflexionar sobre diferentes y nuevas formas de intervención en el ámbito de la discapacidad?

23. ¿Dedica tiempo a conocer los intereses de la persona con DI de cara a desarrollar las diferentes actividades del día?

24. ¿Ha recibido formación específica sobre la PCP? Responda según $1=$ Nada, 2= Poco, 3=Algo, 4= Bastante, 5= Mucha.

¿De qué tipo?

25. Si se propusiera como objetivo educativo desarrollar la PCP señale cuál de las siguientes dificultades obstaculizan su consecución

- Falta de tiempo

- Falta de formación

- Falta de recursos y de apoyo institucional

- Dificultad para diseñar actividades con estos contenidos

- Irrelevancia del tema para la población con DI

- La propia limitación cognitiva de la persona con DI

- Falta de concienciación sobre la importancia del tema 